\title{
APPLICATION OF THE CATALYST KI-MCM-41 IN THE OTERTIC OIL ESTERIFICATION REACTION (licania rigida Benth)
}

\author{
PEREIRA, Marília Gabriela Araújo ${ }^{*}$, SILVA, Antônio Alex de Lima²; MATIAS, Luiz Gonzaga \\ de Oliveira ${ }^{3} ;$; CALDEIRA, Vinicius Patrício da Silva 3 ; SANTOS, Anne Gabriella Dias; \\ SOUZA, Luiz Diं
}

${ }^{1}$ Universidade Estadual do Rio Grande do Norte, Departamento de Química, Faculdade de Ciências Exatas de Naturais, Av. Prof. Antônio Campos, cep. 59625-620, Mossoró - RN, Brasil

(fone: +55 84996667626 )

2 Universidade Estadual do Rio Grande do Norte, Programa de Pós-Graduação em Ciências Naturais, Av. Prof. Antônio Campos, cep. 59625-620, Mossoró - RN, Brasil

(fone: +55 8488388764 )

${ }^{3}$ Universidade Estadual do Rio Grande do Norte, Departamento de Química, Faculdade de Ciências Exatas de Naturais, Av. Prof. Antônio Campos, cep. 59625-620, Mossoró - RN, Brasil

\author{
${ }^{*}$ Autor correspondente \\ e-mail: marilia-gap@hotmail.com
}

Received 09 November 2018; received in revised form 23 December 2018; accepted 04 January 2019

\section{RESUMO}

O biodiesel é um combustível renovável constituído por ésteres monoalquilicos de ácidos graxos, podendo ser produzidos por óleos vegetais, gorduras animais e até óleos residuais derivados da indústria alimentícia. No entanto, a oleaginosa mais usada para produção é a soja, sendo necessário a busca por oleaginosas que não entrem na competição do mercado alimentício. Um estudo sobre oleaginosas se faz necessário para avaliar as propriedades físico-químicas. Nesse sentindo a oiticica se apresenta como uma espécie típica de matas ciliares da caatinga verdadeira. Sua amêndoa constitui cerca de $70 \%$ do fruto e contém de 60 a $63 \%$ de óleo que em sua composição química, apresenta, principalmente, os ácidos graxos licânico (70 a $80 \%$ ) e o linolênico (10 a 12\%). O trabalho analisou as propriedades físico-químicas do óleo de oiticica extraído da semente verde e comparou com as das sementes maduras disponíveis na literatura. $\mathrm{O}$ biodiesel da semente verde foi produzido usando como catalisador o MCM-41 impregnado com iodeto de potássio (KI) nas concentrações 2,5 e $7,5 \%$. A impregnação do $\mathrm{KI}$ desorganizou ligeiramente a estrutura e alterou as propriedades texturais do MCM-41 aumentando o diâmetro de poro e diminuindo o volume de poros e a área superficial, porém os principais picos típicos do MCM-41 não foram destruídos. Antes da aplicação foi realizada a hidrólise do óleo para a eliminação dos materiais insaponificaveis e em seguida, a esterificação do ácido graxo com diferentes proporções de KI. O resultado foi conversão dos ácidos graxos, em ésteres, ocorrendo também a formação de materiais de maior massa molar. Dessa forma nota-se que óleo da semente verde comparado com a madura possui propriedades distintas, sendo necessário o pré tratamento para a reação de biodiesel e que os catalisadores usados proporcionam porcentagens distintas de conversão em ésteres metílicos.

Palavras-chave: Oiticica, semente verde, catalise heterogênea, MCM-41

\section{ABSTRACT}

The Biodiesel is renewable fuel constituted for monoalkyls esters of fatty acids, can be produced by vegetable oil, animal fat and even residual oils from food industry. However the most used oleaginous to produce is soy, being necessary search for another king of oleaginous that do not enter the food market competition. A research about oleaginous is necessary to evaluate the physical-chemical properties. The 
Oiticica Tree is a typical species from riparian forests of the caatinga. Its fruit is composed $70 \%$ for a nut and content between 60 and $63 \%$ of oil in its chemical composition, presently mainly the licorice fatty acids (70 up to $80 \%$ ) and linolenic (10 up to $12 \%$ ). This study analyzed the physicochemical properties of the extracted oil from Oiticica Tree green nut in comparison with extracted oil from mature nuts in available studies. The Biodiesel from green nut was produced using as catalysts MCM-41 impregnated with potassium iodide (KI) in the concentrations 2,5 and $7,5 \%$. The impregnation with $\mathrm{KI}$ slightly disorganized the structure and altered the textural properties of MCM- 41 increasing the pore diameter and reducing pore volume and surface area, but the main typical peaks of MCM-41 were not destroyed. Before the application, the oil was hydrolyzed for the elimination of unsaponifiable materials after that a fatty acid esterification with different proportions of KI. The result was conversion of fatty acids preferably in acid esters, but also had the formation of materials of higher molar mass not identified in this study. In this way we can see that the oil from green seed in comparation with matures seed have different properties being necessary the pre-treatment for biodiesel reaction and the used catalysts provides different percentages of conversion to methyl esters.

Keywords: Oiticica, green seed, heterogeneous catalysis, MCM-41

\section{INTRODUÇÃO}

O biodiesel é um combustível renovável e não-poluente, que pode substituir o óleo diesel de origem fóssil (Paulillo et al. 2007). Apresenta vantagens ambientais em relação ao diesel mineral, como baixos níveis de emissão de monóxido de carbono, não liberação de compostos de enxofre na atmosfera e menores emissões dos principais gases do efeito estufa, onde 0 gás carbônico produzido na sua combustão é quase que totalmente fixado durante o cultivo das oleaginosas (Peterson et al. 1998; Zhang et al. 2011). O biodiesel possibilita a geração de empregos no campo, devido ao desenvolvimento da agricultura nas zonas rurais, a enorme área agricultável e a grande quantidade de plantas produtoras de óleos no Brasil são consideradas como vantagens na corrida científica pelo domínio dessa nova tecnologia. No entanto, o óleo de cada planta é diferente em suas características básicas como composição e estrutura química o que reflete em propriedades físico-químicas diferentes, e consequentemente, também, produz biodieseis com propriedades diferentes, o que dificulta o controle de qualidade do combustível comercializado (Pinto et al. 1963).

No Nordeste, algumas culturas se destacam por serem adaptáveis ao clima do semiárido como é o caso da mamona, pinhão manso e oiticica (Monteiro, 2007; Cordeiro, et al. 2011). A oiticica (Licania rigida Benth), da família Chrysobalanaceae, é uma espécie produtora de óleo que tem aplicações industriais na indústria de tintas e secantes e de sabões (Oliveira et al. 2012). O biodiesel pode ser produzido pela transesterificação, também conhecida como alcoolize, de óleos vegetais e/ou gorduras animais ou hidroesterificação, a qual consiste na hidrólise dos triacilglicerois, seguida da esterificação dos ácidos graxos. A vantagem deste processo reside na possibilidade da utilização de matérias-primas de menor valor agregado, independentemente de sua acidez e umidade. Isso evita problemas de contaminação do biodiesel com resíduos de glicerol livre ou total (mono, di e triacilglicerois), gerando-se um produto de alta qualidade e uma glicerina com elevado grau de pureza (Silva, 2009).

Atualmente a produção industrial de biodiesel ocorre através da transesterificação utilizando a catalise básica homogênea, tendo como vantagens o alto rendimento de conversão e baixo tempo (Helwani, et al. 2009; Cordeiro et al. 2011). Porém, a difícil separação do catalisador, produção de sabões, e geração de muito efluente residual poluidor, são desvantagens dessa rota, (Gallo, 2005). Por tais motivos vêm-se procurando formas mais limpas, seguras, rápidas e eficientes para produção de biodiesel. Com isso os catalisadores heterogêneos têm tido grande interesse das áreas científicas e industriais, pois oferecem vantagens como: fácil manuseio, recuperação e reutilização do catalisador, além de gerar menos resíduos (Soldi et al. 2009; Ghesti, et al. 2012).

Os catalisadores básicos heterogêneos apresentaram taxas de conversões maiores do que os sólidos ácidos e, desta forma, têm sido estudados com maior ênfase. Alguns trabalhos relatam a obtenção de catalisadores mesoporosos pela incorporação de óxidos alcalinos e alcalino-terrosos, ou moléculas 
orgânicas básicas, na superfície dos materiais mesoporosos (Tapanes, et al. 2008; Tapanes, Perez, Cruz, 2013; Cantrel et al. 2005; Wang et al. 2005). Em contrapartida, a aplicação de tais compostos como catalisadores heterogêneos na síntese de biodiesel apresenta problemas, devido à fácil carbonatação e lixiviação parcial dos mesmos no meio reacional (Galvão et al. 2012).

Uma das maneiras de conter tais problemas é incorporar o óxido em um suporte catalítico. Neste sentido, a peneira molecular mesoporosa do tipo MCM-41, se apresenta como um suporte catalítico favorável para o desenvolvimento de novos catalisadores, com fases ativas e bem dispersas. Ela apresenta um arranjo hexagonal de mesoporos unidimensionais com diâmetro que pode variar entre 2-10 nm, uma estabilidade térmica adequada, volume de poros alto $e$ área superficial acima de $700 \mathrm{~m}^{2} \mathrm{~g}^{-1}$, além da acessibilidade á moléculas grandes (triglicerídeos e ácidos graxos) aos sítios ativos no interior dos poros, estas características vêm tornando o MCM-41 um material bastante promissor na catálise (Castro, 2010).

Galvão et al. 2012, mostram um trabalho em que iodeto de potássio é suportado em peneiras moleculares mesoporosas (SBA-15 E MCM-41) como catalisador básico para síntese de biodiesel no qual as taxas de conversões são consideráveis, em um tempo de reação muito menor, se comparados com outras reações que envolvem iodo (Ramalinga et al. 2002; Li e Xie, 2006).

Com base nas principais problemáticas abordadas, este trabalho teve como objetivo estudar as características físico-químicas do óleo de oiticica extraído da semente verde e avaliar a atividade catalítica de catalisadores impregnados com diferentes porcentagem de $\mathrm{KI}$ (iodeto de potássio), na produção de biodiesel via hidroesterificação

\section{MATERIAL E MÉTODOS}

\subsection{Coleta, tratamento e extração do óleo de oiticica}

A coleta das sementes de oiticica (Licania rigida Benth) foi realizada na comunidade de Lagoa de Paus, em fevereiro de 2014 na zona rural de Mossoró (RN). As sementes foram extraídas verdes, lavadas e secas em estufa a $60^{\circ} \mathrm{C}$ por 96 h. Posteriormente foram descascadas e trituradas mecanicamente em liquidificador industrial. A extração do óleo foi realizada a quente, em sistema soxhlet com nhexano por um período de $4 \mathrm{~h}$. O óleo foi purificado através da sua concentração num evaporador rotativo a pressão reduzida (Oliveira et al. 2012).

\subsection{Reação de hidroesterificação}

Com finalidade de obter conversões melhores realizou-se uma hidrólise seguida de uma esterificação. A hidrólise do óleo foi realizada numa razão molar de 1:3 de óleo: hidróxido de sódio, utilizando metanol como solvente e 3\% de catalisador. Estas foram realizadas em um balão de fundo chato de 250 $\mathrm{mL}$, acoplado a um condensador em refluxo e um agitador magnético, sob pressão atmosférica, durante um período de $3 \mathrm{~h}$. Após a reação, a mistura foi colocada em um funil de separação, separando-se o glicerol e a água do meio. Os ácidos graxos foram submetidos ao processo de esterificação. Para tal processo utilizou-se os ácidos graxos obtidos na razão molar 1:1 com metanol, utilizando para cada reação $3 \%$ dos catalisadores sintetizados. A reação foi realizada em um balão de vidro de $250 \mathrm{ml}$ acoplado a um condensador, em refluxo e um agitador magnético sob pressão atmosférica, durante 6 horas de reação. Após a reação foi feita a purificação do biodiesel de maneira semelhante ao usado na transesterificação (Ranucci, C. R., et al. 2015)

\subsection{Caracterizações realizadas no óleo de Oiticica}

No óleo de oiticica foram realizadas as análises de densidade (por picnômetria), índice de acidez, índice de saponificação, água e sedimentos, ácidos graxos livres, índice de iodo, índice de peróxido, viscosidade cinemática e ponto de fulgor/combustão seguindo normas padrões (Santos, 2009; Oliveira, et al. 2012). Após a reação de biodiesel com o intuito de obter as principais características do biodiesel formado, foram realizadas as análises térmicas das amostras, o índice de acidez e e iodo, seguindo os procedimentos utilizados por Santos, (2009) e Oliveira, et al. (2012).

\subsection{Síntese do MCM-41 e Impregnação do $\mathrm{KI}$ ao material}

A síntese do catalisador MCM-41 foi proposto por Araújo et al. (2005), utilizando a 
proporção entre os reagentes de 1,0 CTMABr. $1,0 \mathrm{NaOH} .4,0 \mathrm{SiO}_{2} .200,0 \mathrm{H}_{2} \mathrm{O}$. Inicialmente, a síntese se processa com o uso de duas soluções preparadas separadamente. A figura 1 esquematiza a rota utilizada na síntese do MCM-41.

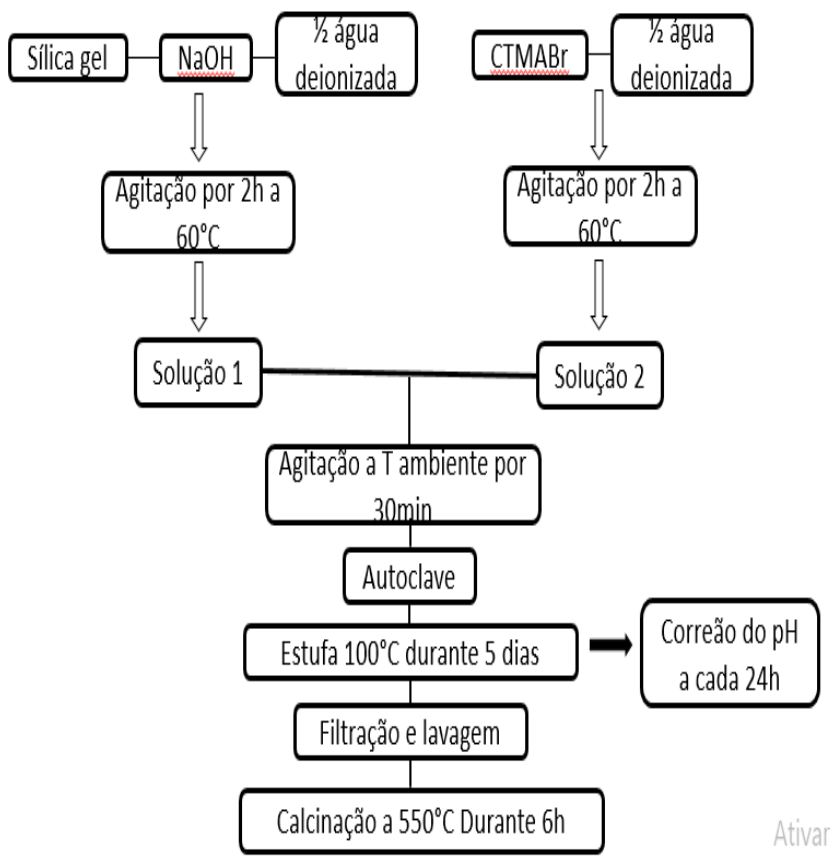

Fonte: autor, 2018.

Figura 1. Esquema de representação da síntese do MCM-41

\subsubsection{Impregnação do material}

As impregnações foram feitas com diferentes concentrações de $\mathrm{KI}$ sendo elas: 2,5 e $7,5 \%$. Estes suportes foram impregnados pelo método via excesso de solvente com soluções aquosas de iodeto de potássio. Para tanto, 50 $\mathrm{mL}$ das soluções diluídas de iodeto de potássio, nas respectivas \% em peso, foram misturados a $1,0 \mathrm{~g}$ de suporte. Em seguida, esse sistema teve sua água reduzida por meio de agitação, a $80^{\circ} \mathrm{C}$, sob pressão ambiente durante 30 minutos. Em seguida o material foi seco em estufa a $100 \stackrel{\circ}{\circ}$. Posteriormente o sólido impregnado foi calcinado a $500 \stackrel{\circ}{\circ}$ sob fluxo de ar $\left(100 \mathrm{~mL} \mathrm{~min}^{-1}\right)$ por $3 \mathrm{~h}$.

\subsubsection{Caracterização realizadas nos catalisadores obtidos (MCM-41 e \%KI-MCM-41)}

\subsubsection{Difração de raios- $X$}

Para confirmar se a estrutura típica dos materiais MCM-41 e a possível formação de óxidos no material, bem como alteração das estruturas causadas pela incorporação do KI se usou a técnica de difração de Raios- X. Foi utilizado o equipamento Rigaku modelo Mini Flex II, usando radiações de CuKa, em uma voltagem de $30 \mathrm{k} / \mathrm{V}$ e corrente de tubo de $30 \mathrm{~m}$ em baixo ângulo (20 de $1^{\circ}$ a $10,0^{\circ}$.). Foi utilizado o método do pó, sendo as amostras previamente pesadas para então serem analisada

\subsubsection{Adsorção e dessorção de $N_{2}$ (BET e BJH)}

A análise de Adsorção/dessorção de Nitrogênio foi realizada utilizando os métodos BET e BJH para estimar, a área superficial específica, volume de poro total dos catalisadores e diâmetro de poros e assim poder relacionar estas propriedades do MCM-41 puro com as mesmas propriedades nos catalisadores com o KI impregnado. O equipamento utilizado foi o modelo ASAP 2020 da Micromeritics. Antes de cada análise, os materiais foram, previamente, desgaseificados, por 10 horas a $300^{\circ} \mathrm{C}$ a vácuo. Após a desgaseificação, as amostras foram submetidas à adsorção de nitrogênio a $77 \mathrm{~K}$ para obtenção das isotermas, as quais foram realizadas na faixa de pressão relativa $(\mathrm{P} / \mathrm{Po})$ entre 0,0001 e 0,99.

\subsubsection{Termogravimetria (TG/DTG)}

A análise termogravimétrica (TG/DTG) foi realizada nos catalisadores com 0 intuito, principal, de acompanhar as perdas de massa das amostras de catalisador para se determinar a melhor temperatura para a calcinação e nos produtos da reação de esterificação para analisar a conversão de óleo em biodiesel. Para isso , cerca de $5 \mathrm{mg}$ das amostras foram submetidas a um aquecimento de 25 a $900^{\circ} \mathrm{C}$ para o catalisador e de 25 a $800^{\circ} \mathrm{C}$ para biodiesel. Para ambos os processos se utilizou, uma razão de aquecimento de $10 \% / \mathrm{min}$, sob atmosfera de nitrogênio com fluxo de $70 \mathrm{~mL} \cdot \mathrm{min}^{-1}$. O equipamento utilizado foi STA 449 jupiter da Netzsch.

\section{RESULTADOS E DISCUSSÃO:}

\subsection{Extração, Caracterização e Teor do Óleo de Oiticica}

Segundo Oliveira, et al. (2012) as sementes de oiticica madura apresentam teor de óleo $65 \%$, sendo que o teor obtido para as sementes verdes neste trabalho foi de aproximadamente $35 \%$. Esse resultado mostra que a semente verde tem, aproximadamente, 
$50 \%$ a menos de óleo do que as sementes maduras. Mesmo assim, a semente verde já contém uma quantidade substancial de triglicerídeos, se comparada à outras oleaginosas, como, algodão (15\%) e a soja (18\%) que, atualmente, são utilizados na produção comercial de biodiesel (Araújo e colaboradores, 2010 (a). A tabela 1 mostra as propriedades físico-químicas do óleo extraído da semente verde em comparação com as mesmas propriedades do óleo da semente madura (Oliveira, et al. 2012), indicando diferenças notórias na qualidade dos óleos.

Tabela 1: Caracterizações físico-químicas dos óleos extraído das sementes verdes e maduras de oiticica.

A análise do índice acidez mostra que a acidez do óleo da semente verde é menor que o da semente madura. Apesar da sua acidez ainda estar elevada, se comparadas com oleaginosas como soja, algodão (Araújo et al. 2010 (B)) que possuem acidez mais baixa. Uma acidez alta indica uma intensa presença de ácidos graxos livres, e neste caso a matéria-prima deve passar por um tratamento prévio, para poder ter uma boa aplicação em transesterificações básicas. Essa alta acidez é confirmada no alto valor obtido para a análise de ácidos graxos livres ( $A G L)$, indicando que o óleo tem ácidos graxos livres em grande quantidade. Isso deixa claro a necessidade da realização de um procedimento de tratamento do óleo como hidrólise seguida de esterificação ou aumento da quantidade de catalisador para a produção de biodiesel. Apesar da acidez mais alta no óleo da semente madura, - AGL é menor indicando que o processo de amadurecimento altera a composição do óleo.

O índice de saponificação da semente verde é superior ao da madura, o que mostra que esta possui mais compostos de menor massa molecular conforme indicado pelo valor de AGL. Este resultado indica que a formação de compostos de maior massa molecular ocorre com o amadurecimento da semente.

O índice de iodo está diretamente relacionado com o total de insaturações de um material lipídico, ou seja, o índice de iodo aumenta com 0 aumento de insaturações (Bouaid et al. 2007). O valor alcançado para ambos os óleos indica a presença de duplas conjugadas, advindo do seu principal ácido graxo, o licânico. Foi notado que o óleo bruto tem predisposição à solidificação, em temperatura ambiente torna-se mais viscoso e em menores temperaturas separa-se em duas fazes de diferentes densidades e aspecto físico.

As viscosidades de óleos de oleaginosas possuem valores elevados, o que já é esperado, uma vez que apresentam cadeias grandes e de elevadas massas moleculares que facilitam o entrecruzamento e dificultam o movimento (Alptekin; Canakci, 2008). Não foi diferente com a oiticica, com o óleo sendo muito viscoso, o que pode complicar a reação de transesterificação, por dificultar a homogeneização, o que em geral é resolvido aumentando a razão óleo/álcool e fazendo reação heterogênea ou ácida.

Os resultados de densidade estão de acordo com os de índice de acidez e indicam que os compostos presentes no óleo das sementes verdes são menores e se empacotam melhor produzindo um óleo mais denso.

Os pontos de fulgor e de combustão confirmam as propriedades discutidas acima. Para estas propriedades quanto maior for o peso molecular, maior será a energia necessária para produzir uma faísca (fulgor) ou para entrar em combustão. Cabe ressaltar que valores muito elevados implicam em dificuldade maior na queima do combustível e trabalho do motor sob temperaturas mais altas, o que poderia refletir em maior desgaste das peças dos automóveis (Santos, 2010). Ambos os óleos estão isentos de água e sedimentos, o que é relevante, pois mostra que o pré-tratamento das sementes foi eficiente.

Através dos dados termogravimétricos do óleo da semente verde, pode-se observar na figura $2 \mathrm{~A}$ (preto) que ocorreu uma única perda de massa referente à degradação/volatilização das misturas de ácidos graxos presente no óleo. A curva de DTG do óleo (3B preto) mostra um alargamento e a existência de um segundo evento térmico (ombro) que se sobrepõe ao pico principal,indicando a presença de compostos com diferentes funções químicas e ou tamanho de cadeia carbônica, ou seja, diferentes massas molares (MM). Por outro, os resultados das análises físico-químicas (acidez e viscosidade altas) sinalizavam para a necessidade de tratamento do óleo, assim realizou-se a sua hidrolise e repetiu-se as análises. Após hidrolise nota-se que a maior parte do material resultante possui uma temperatura de degradação/volatilização menor (ver figuras 2A e 2B), mas tem, também compostos com maior massa molar, o que indica que tem-se uma 
mistura de ácidos graxos com pelo menos 4 compostos diferentes (perdas de massa com picos em $255^{\circ} \mathrm{C}, 350^{\circ} \mathrm{C}, 450^{\circ} \mathrm{C}$, e região larga acima entre $500^{\circ} \mathrm{C}$ e $700^{\circ} \mathrm{C}$ ), sendo que cerca de $5 \%$ deles só volatilizam acima $500^{\circ} \mathrm{C}$ e $15 \%$ entre $400^{\circ} \mathrm{C}$ e $500^{\circ} \mathrm{C}$ o restante aproximadamente $60 \%$ se volatiliza em aproximadamente $255^{\circ} \mathrm{C}$ e corresponde a maior quantidade de material.

Assim a esterificação foi feita com esta mistura de ácidos graxos obtida com a hidrólise do óleo de oiticica.

\subsection{Caracterização dos catalisadores MCM- 41, KI-MCM-41-2,5\% e KI-MCM-41-7,5}

Após a síntese do MCM-41 realizou-se a analise termogravimétrica com o intuito de saber a temperatura de calcinação. Ao analisar a figura 3(A) nota-se três perdas que se destacam, a primeira perda em torno da temperatura de $100^{\circ}$ é referente, a perda água ou umidade no material, a segunda entre $200^{\circ} \mathrm{C}$ e $330^{\circ} \mathrm{C}$ é referente a saída do direcionador orgânico e a terceira que se estende de $330^{\circ} \mathrm{C}$ a $600^{\circ} \mathrm{C}$ que também é devida a saída lenta de catalisador orgânico. Com base nessa analise realizou-se a calcinação a $550^{\circ} \mathrm{C}$ e o material resultante foi submetido a nova análise de forma a certificar a total retirada do direcionador orgânico. Como é mostrado na figura 3(B) não aparecem os eventos referente a saída do direcionar o que mostra a sua total retirada da estrutura.

No que se diz respeito a síntese do MCM41 , analise de difração de raio- $X$ mostra que o catalisador apresenta os três picos de difração referentes aos planos de difração (100), (110) e (200), característicos do arranjo hexagonal de mesoporos unidimensionais da estrutura do MCM-41 (Figura 4)

A figura 5 apresenta os difratogramas de raio- $x$ para os materiais impregnados com diferentes proporções de $\mathrm{KI}$ no catalisador mesoporoso MCM-41. Nota-se que a estrutura inicial do material é desorganizada, fato indicado pela ausência dos picos 110 e 200 e pelo deslocamento do pico principal para valores de $2 \theta$ maiores em relação ao catalisador puro, fato já registrado na literatura quando ocorre a inserção de materiais na estrutura de materiais mesoporosos. (Galvão et al. 2012). No entanto, a presença do pico principal (100) com forte intensidade indica que a mesma não foi destruída. Também se observa, que a medida que se insere $\mathrm{KI}$ ocorre alterações na intensidade do pico principal, que diminui com o aumento de $\mathrm{KI}$, confirmando que ocorre desorganização da estrutura.

As caracterizações dos materiais via absorção/dessorção de $\mathrm{N}_{2}$ (vide figura 6 e tabela 2) confirmam essa modificação e as suas consequências nas propriedades texturais dos catalisadores impregnados.

A análise textural dos materiais impregnados indica que os mesmos possuem isotermas do tipo IVa com histerese do tipo $\mathrm{H} 1$ encontrada em materiais que exibem uma faixa estreita de mesoporos uniformes. As propriedades variam, de forma geral de acordo com o aumento da porcentagem de $\mathrm{KI}$ inserido, aumentando o tamanho dos poros e diminuindo o volume e a área específica como mostra a figura 7 e a tabela 2. Estes dados podem ser resultado do entupimento dos poros com o aumento de $\mathrm{KI}$ no suporte que causa uma deformação dos poros.

\section{Tabela 2. Propriedades texturais dos catalisadores s obtidos}

\subsection{Caracterizações realizadas nos biodieseis}

As figuras $8 \mathrm{~A}$ e $\mathrm{B}$ mostram os resultados sobrepostos de TG e de DTG para o óleo de oiticica, os produtos obtidos nas reações de esterificação com 2,5 e com 7,5\% de $\mathrm{KI}$, para o produto da transesterificação e repete a curva para a mistura de ácidos graxos obtida do óleo da oiticica (Figura 2) com as suas 4 perdas de massas já discutidas. $\mathrm{Na}$ tabela 3 são observadas as temperaturas, as perdas de massas e o material residual para os materiais analisados.

Comparando as curvas de DTG do óleo com as curvas do ácido graxo obtido observa-se que o óleo tem três compostos, os quais apresentavam perda de massa em baixas temperaturas, 250 a $300{ }^{\circ} \mathrm{C}(3 \%$ de perda), em $395(95 \%)$ e uma pequena perda de $2 \%$ (ombro do pico principal) na temperatura de $450{ }^{\circ} \mathrm{C}$. A hidrólise retira compostos que se situam na faixa de 150 a $350^{\circ} \mathrm{C}(70 \%$ de perda) e compostos de maior massa molar na faixa de 350 a $400^{\circ} \mathrm{C}$ (10\% de perda), de 400 a $510^{\circ} \mathrm{C}(17 \%$ de perda) e de 510 a $650^{\circ} \mathrm{C}$ (3\% de perda). A faixa de perda massa do pico principal (pico e ombro) tem sido identificada na literatura como sendo devido a degradação/volatilização de ácidos graxos. A comparação das curvas do óleo e dos ácidos 
graxos com as curvas do material transesterificado ou esterificado mostram claramente que a transesterificação e a esterificação ocorrem, o que é indicado pelo aparecimento de picos em temperaturas bem menores que as temperaturas do óleo $\left(395^{\circ} \mathrm{C}\right)$ para a transesterificação $\left(251^{\circ} \mathrm{C}\right)$ e temperaturas ligeiramente menores que a dos ácidos graxos $\left(255^{\circ} \mathrm{C}\right)$ para os produtos de esterificação $\left(245^{\circ} \mathrm{C}\right)$.

Tabela 3. Perdas de massa das amostras transesterificadas, esterificadas e materiais de partida

O segundo pico (região de $350^{\circ} \mathrm{C}$ ) aparece mais claro e, também, ligeiramente deslocado para a esquerda se comparado com o ácido graxo, indicando que, também, pode ser devido a biodiesel com cadeias carbônicas maiores e ou com estruturas diferentes (duplas ligações), o que é coerente com a existência de compostos de maior MM nos ácidos graxos e com o fato deste óleo se separar em duas fases na temperatura ambiente. $\mathrm{O}$ aumento de $\mathrm{KI}$ diminui a \% de material perdido nessa faixa e causa o aparecimento de duas perdas de massa nas faixas de temperatura de $266-317^{\circ} \mathrm{C}$ e 317 - $379^{\circ} \mathrm{C}$. O terceiro pico continua existindo, mas ao contrário dos anteriores não altera sua posição (aproximadamente $450^{\circ} \mathrm{C}$ ) com nenhuma das reações realizadas (hidrólise, transesterificação e esterificação) o que indica que são compostos já existentes no óleo e que são inertes e permanecem na mistura sem reagir.

O quarto pico continua existindo na mesma faixa de temperaturas $\left(500\right.$ a $\left.700^{\circ} \mathrm{C}\right)$, mas a sua intensidade é aumentada com o aumento da porcentagem de $\mathrm{KI}$, indicando que esse material pode dar características ao catalisador de formar ligações de maior massa molar. O aumento da quantidade de $\mathrm{KI}$ para $7,5 \%$ proporciona um evento adicional na região próxima a $260^{\circ} \mathrm{C}$, diminui a quantidade de material perdido no pico principal e aumenta a quantidade de material próximo de $330^{\circ} \mathrm{C}$ e na região de alta temperatura, além de aumentar muito a quantidade de material de alta MM. Assim o KI atua facilitando a quebra de cadeias na dupla ligação e posterior ligação dessas cadeias formando moléculas de maior MM.

Estes resultados são coerentes com a diminuição dos índices de iodo e de acidez medidos no óleo, ácido graxo e produtos de esterificação (tabela 4) e indicam que está ocorrendo além da esterificação, também, a quebra de cadeias dos ácidos graxos que, posteriormente, voltam a se ligar formando cadeias maiores, o que aumenta a temperatura de volatilização no qual o $\mathrm{KI}$ age catalisando este processo e aumento da quantidade de compostos de maior MM.

\section{Tabela 4. Caracterizações realizadas no biodiesel}

As porcentagens de resíduos confirmam
estes resultados e mostram que a transesterificação do óleo bruto (3,69\%) aumenta a porcentagem de resíduos $(4,98 \%)$ devido a formação de compostos de alta $\mathrm{MM}$ não volatizáveis durante o próprio experimento e a existência de compostos de alta MM no óleo. A hidrólise limpa o óleo desses compostos de alta MM e produz compostos de 4 tamanhos de MM, todos totalmente volatizáveis, não produzindo nenhum resíduo (0\%). A esterificação com 2,5\% de $\mathrm{KI}$ produz 2,04\% de resíduos indicando que 0 $\mathrm{KI}$ em pequenas quantidades catalisa a formação de compostos de alta MM, mas o aumento de $\mathrm{KI}$ $(7,5 \%)$ reduz novamente os resíduos a zero, indicando que ela também catalisa a quebra de compostos de alta MM em compostos menores. Resultados semelhantes desta variação da MM com 0 catalisador foram obtidos em experimentos de termogravimetria de óleo de palma (Santos et al. 2014) tratados por diferentes tempos em diferentes temperaturas, indicando que essas quebras e formação de ligações devem ocorrer no óleo em função de variáveis como tempo ou temperatura.

Assim, o produto final obtido nas esterificações, provavelmente é uma mistura composta em sua maioria (aproximadamente $90 \%$ para $2.5 \%$ de $\mathrm{KI}$ e $82 \%$ para $7,5 \%$ de $\mathrm{KI}$ ) de uma mistura de diferentes ésteres e de outros compostos não identificados de alta massa molar que já existiam no óleo inicial ou foram formados durante o experimento devido à quebra e formação de novas ligações no material. O catalisador $\mathrm{KI}$ atua facilitando a aumento da quantidade de compostos de alta MM que ocorre durante o experimento. 
CONCLUSÕES:

As impregnações realizadas não causaram a destruição da estrutura mesoporosa do MCM-41. As propriedades físico-químicas do óleo da semente verde e madura são de qualidades distintas e indicam que o óleo deve ser purificado antes de ser esterificado. O KI catalisa a reação de esterificação nas duas proporções de catalisador usada, sendo que foi observado que, além da esterificação que é a reação principal, ocorre quebras e formação de novas cadeias, 0 que altera a constituição/composição do produto obtido com a variação do $\mathrm{KI}$, o que é indicado pelas alterações tanto das medidas físico-químicas (índice de acidez e de iodo) quanto pelas analises térmicas. Observa-se que o aumento $\mathrm{da} \%$ de $\mathrm{KI}$ provocou um aumento da massa molar dos compostos e aumentou a fração deles na mistura, diminuindo a quantidade de ésteres formados de aproximadamente $90 \%$ para $82 \%$ respectivamente

\section{AGRADECIMENTOS:}

Ao CNpq pelo fomento da bolsa.

Ao Laboratório de Catalise Ambiente e Materiais (LACAM) da UERN.

\section{REFERÊNCIAS:}

1. Beltrão; N.E.M. E. OLIVEIRA; M.I.P. N.177, Oleaginosas potencias do nordeste para produção de biodiesel, Documentos MAPA Campina Grande PB, 2007.

2. Cantrell D.G., Gillie Lisa J., Lee Adam F., Wilson Karen., Structure reactivity correlations in $\mathrm{MgAl}$ hydrotalcite catalysts for biodiesel synthesis Appl. Catal. A: 2005 Gen. 287, 183-190.

3. Castro, K.K.V. Sintese, caracterização e aplicação do MCM-41 E Al-MCM-41 na pirolise do resíduo atmosférico de petróleo, dissertação de mestrado, UFRN, Natal-RN, 2010.

4. Cordeiro C. S. et al. Catalisadores heterogêneos para a produção de monoésteres graxos (biodiesel) Quim. Nova, 2011, Vol. 34, No. 3, 477-486, Paraná.
5. Di serio, M.; Tesser, R.; Pengmei, L.; Santa Cesaria, E.; Energy Fuels 2008, 22, 207.

6. Gallo, J. M. R. Síntese e caracterização de $[\mathrm{Nb}]-\mathrm{MCM}-41$ e $\mathrm{NbxOy}(\mathrm{OH}) \mathrm{z}$ Montmorilonita e aplicações em catalise redox e ácida. Dissertação de mestrado, UNICAMP, São Paulo 2005

7. Galvão, L. P. F. C. et al. lodeto de potássio suportado em peneiras moleculares mesoporosas (SBA-15 e MCM-41) como catalisador básico para síntese de biodiesel Quim. Nova, Vol. 35, No. 1, 41-44, 2012.

8. Garcia, C. M. et al. Transesterification of soybean oil catalyzed bysulfated zirconia.; Bioresour. Technol., 99, 6608, 2008.

9. Ghesti, G. F. et al. Produção de biodiesel via transesterificação etílica com zeólitas básicas Quim. Nova, Vol. 35, No. 1, 119123, 2012.

10. Helwani Z. et al. Solid heterogeneous catalysts for transesterification of triglycerides with Applied Catalysis A: General 3632009 1-10.

11. Li, H.; Xie, W. Transesterification of soybean oil to Biodiesel with $\mathrm{Zn} / \mathrm{I2}$ catalyst Catalysis Letters. vol.107, $n^{\circ} 1-2$, 25-30, 2006.

12. Monteiro, J. M. G.; Plantio de Oleaginosas por Agricultores Familiares do Semiárido Nordestino para Produção de Biodiesel como uma Estratégia de Mitigação e Adaptação às Mudanças Climáticas. Tese (Doutorado), Universidade Federal do Rio de Janeiro, Rio de Janeiro, 2007.

13. Oliveira, F. A. G. et al. Síntese, caracterização e avaliação de biodiesel de óleo de oiticica (Licania rigida Benth) e isolalamento do éster metílico do ácido licânico Química: ciência, tecnologia e sociedade Vol. 1, No. 12012 Mossoró$\mathrm{RN}$.

14. Paulillo, L. F.; Vian C. E. de F.; Shikida, P. F. A.; Mello, F. T. Álcool combustível e biodiesel no Brasil: quo vadis? Rev. Econ. Sociol.Rural vol.45 no.3 Brasília July/Sept 2007.

15. Peterson, C. L.; Hustrulid, T.; Biomass and Bioenergy 1998, 14, 91 
16. Pinto, G. P. - Características físicoquímicas e outras informações sobre as principais oleaginosas do Brasil. Recife: Instituto de Pesquisa e Experimentação Agropecuárias do Nordeste. 1963. 83 p. (Boletim Técnico, 18)

17. Ramalinga K.; Vijayalakshmi P.; KaimaL T. N. B. A mild and efficient method for esterification and transesterification catalyzed by iodine. Tetrahedronletters. vol. 43, no5, 879-882, 2002.

18. Rani, V. R.; Kishan, M. R.; Kulkarni, S. J.; Raghavan, K. V., Immobilization of metalloporphyrin complexes in molecular sieves and their catalytic activity Catalysis Communication v. 6, p. 531- 535, 2005.

19. Ranucci, C. R., Alves, H. J., Silva, C., Monteiro, M. R., Andressa, K., Bariccatti, R. A., \& Antonio, E. (2015). Obtaining biokerosene of jatropha (Jatrophacurcas L.) and their mixtures to fossil kerosene. Revista Tecnológica - Edição Especial 2014, 43-52.

20. Soldi R.A. et al. Soybean oil and beef tallow alcoholysis by acid heterogeneous catalysis/ Applied Catalysis A: General 361, 2009, 42-48.

21. Serio, M. Di; et al. Transesterification of Soybean Oil to Biodiesel by Using Heterogeneous Basic Catalysts Ind. Eng. Chem. Res. 2006, 45, 3009.

22. Tapanes, N. A. D. A. G; Perez R. S.; CRUZ Y. R. Biodiesel no brasil: matérias primas e tecnologias de produção, AS\&T V.1, N 1, Rio de Janeiro, 2013.

23. Tapanes, N. C. O. Produção de biodiesel a partir da transesterificação de óleo de pinhão manso (jatropha curcas lin): estudo teórico e experimental, tese de doutorado, UFRJ, Rio de Janeiro-RJ, 2008.

24. Zhang, Y.; Dubé, M. A.; Mclean, D. D.; Bioresour. Technol. 2003, 89, 1. 3. (Quim. Nova, Vol. 34, No. 3, 477-486.

25. Wang, Y. M.; Wu, Z. Y.; Wei, Y. L.; Zhu, J. $\mathrm{H}$. ; Microporous Mesoporous Mater., 84, 127, 2005. 
Tabela 1: Caracterizações físico-químicas dos óleos extraído das sementes verdes e maduras de oiticica

\begin{tabular}{ccc}
\hline Análise & Semente Verde & $\begin{array}{c}\text { Semente Madura } \\
\text { (Oliveira, 2012) }\end{array}$ \\
\hline Teor de óleo $(\%)$ & $35 \%$ & $65 \%$ \\
Índice de acidez $(\mathrm{mg} \mathrm{KOH} / \mathrm{g})$ & 1,39 & 1,53 \\
Ácidos Graxos Livres $(\%)$ & 6,43 & 0,421 \\
Índice de saponificação $(\mathrm{mg} \mathrm{KOH} / \mathrm{g})$ & 198,39 & 162,41 \\
Índice de lodo $(\mathrm{g}$ de iodo $/ 100 \mathrm{~g})$ & 42,435 & 43,58 \\
Índice de peróxido $(\mathrm{mEq} / \mathrm{Kg})$ & 79,27 & - \\
Viscosidade Cinemática $\left(\mathrm{mm}^{2} / \mathrm{s}\right)$ & 23,075 & - \\
Ponto de Fulgor & 231 & - \\
Ponto de combustão $\left({ }^{\circ} \mathrm{C}\right)$ & 315 & 0 \\
Água e sedimentos $(\%)$ & - & 0,949 \\
Densidade $\left(\mathrm{g} / \mathrm{cm}^{3}\right)$ & 0,9515 &
\end{tabular}

Tabela 2. Propriedades texturais dos catalisadores s obtidos

\begin{tabular}{|c|c|c|c|}
\hline Amostras & $\mathrm{Dp}(\mathrm{nm})$ & $V p\left(\mathrm{~cm}^{3} \mathrm{~g}^{-1}\right)$ & $S_{\mathrm{BET}}\left(\mathrm{m}^{2} \mathrm{~g}^{-1}\right)$ \\
\hline MCM- 41 & 2,19 & 0,75 & 902 \\
\hline $2,5 \% \mathrm{KI}-\mathrm{MCM}-41$ & 3,45 & 0,43 & 538 \\
\hline $7,5 \% \mathrm{KI}-\mathrm{MCM}-41$ & 4,03 & 0,32 & 331 \\
\hline
\end{tabular}

Tabela 3. Perdas de massa das amostras transesterificadas, esterificadas e materiais de partida

\begin{tabular}{ccccc}
\hline AMOSTRAS & $\mathbf{T}\left({ }^{\circ} \mathbf{C}\right)$ & $\begin{array}{c}\text { T máx } \\
\left({ }^{\circ} \mathbf{C}\right)\end{array}$ & $\begin{array}{c}\text { PERDA DE } \\
\text { MASSA (\%) }\end{array}$ & $\begin{array}{c}\text { MASSA } \\
\text { RESIDUAL (\%) }\end{array}$ \\
\hline ÓLEO & $240-507$ & 395 & 96,31 & 3.69 \\
\hline TRANSESTERIFICAÇÃO & $107-280$ & 251 & 33,77 & 4,98 \\
& $280-400$ & 349 & 44,45 & \\
& $400-502$ & 428 & 16,92 & - \\
\hline ÁCIDOS GRAXOS & $130-320$ & 255 & 63,15 & \\
& $388-491$ & 439 & 18,46 & \\
\hline Biodiesel 2,5\% & $507-705$ & 584 & 18,39 & \\
KI- MCM-41 & $123-302$ & 245 & 44,52 & \\
& $302-428$ & 352 & 33,13 & \\
& $428-491$ & 449 & 10,75 & \\
\hline Biodiesel 7,5\% & $527-710$ & 623 & 9,81 & \\
KI- MCM-41 & $119-266$ & 245 & 26,28 & \\
& $266-317$ & 284 & 16,85 & \\
& $317-379$ & 339 & 16,70 & \\
& $379-493$ & 438 & 20,60 & \\
& $493-658$ & 573 & 19,71 & \\
\hline
\end{tabular}


Tabela 4. Caracterizações realizadas no biodiesel

\begin{tabular}{ccc}
\hline KI-MCM-41 & INDICE DE ACIDEZ (mg KOH/g) & INDICE DE IODO (g de iodo /100g) \\
\hline Óleo Bruto & 1,39 & 42,43 \\
Ácidos Graxos & 186,26 & 20,52 \\
Reação 2,5\% & 125,14 & 31,70 \\
Reação $7,5 \%$ & 103,24 & 20,52 \\
\hline
\end{tabular}
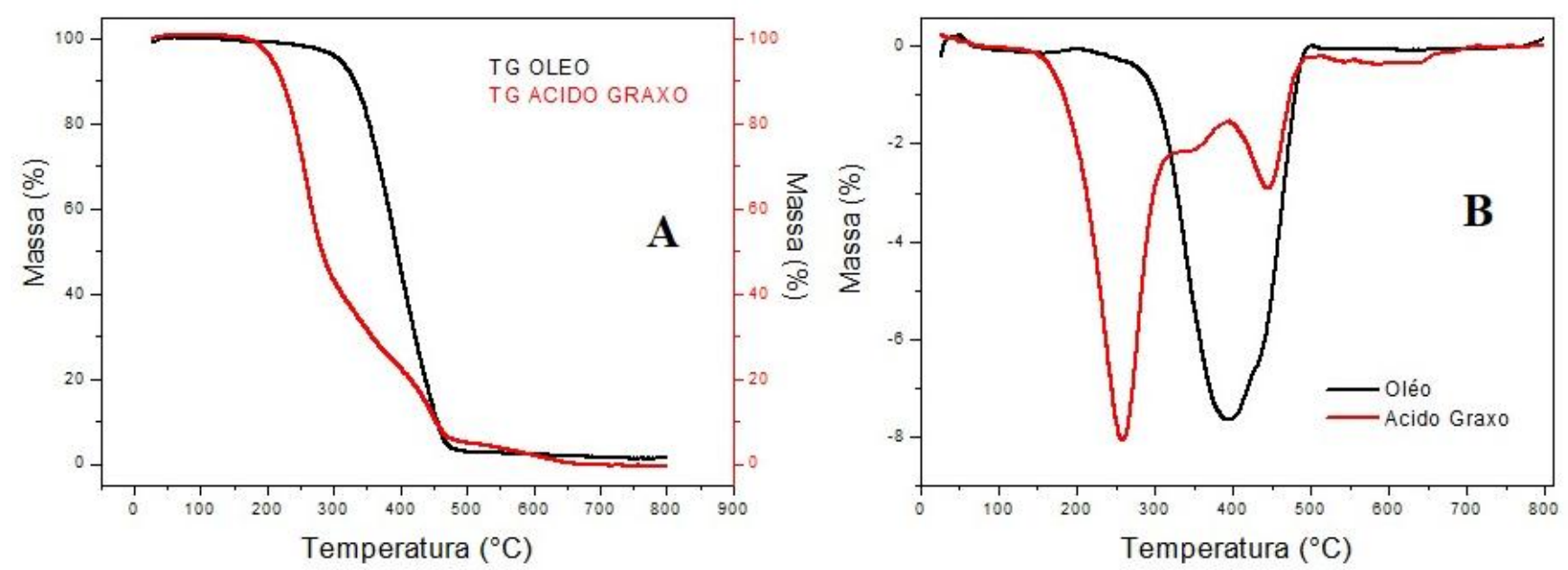

Figura 2. A e B - TG DTG do óleo e ácido graxo de Oiticica
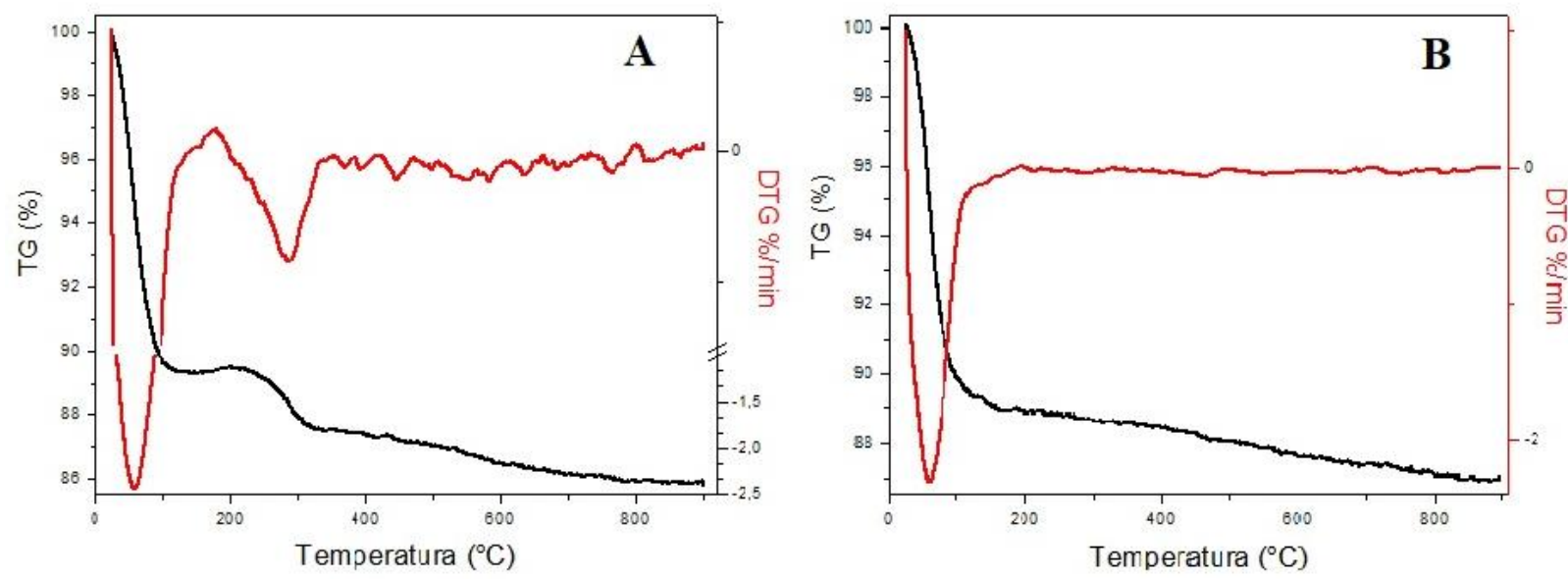

Figura 3. A - TG DTG do MCM-41 não calcinado B - TG DTG do MCM-41 calcinado 


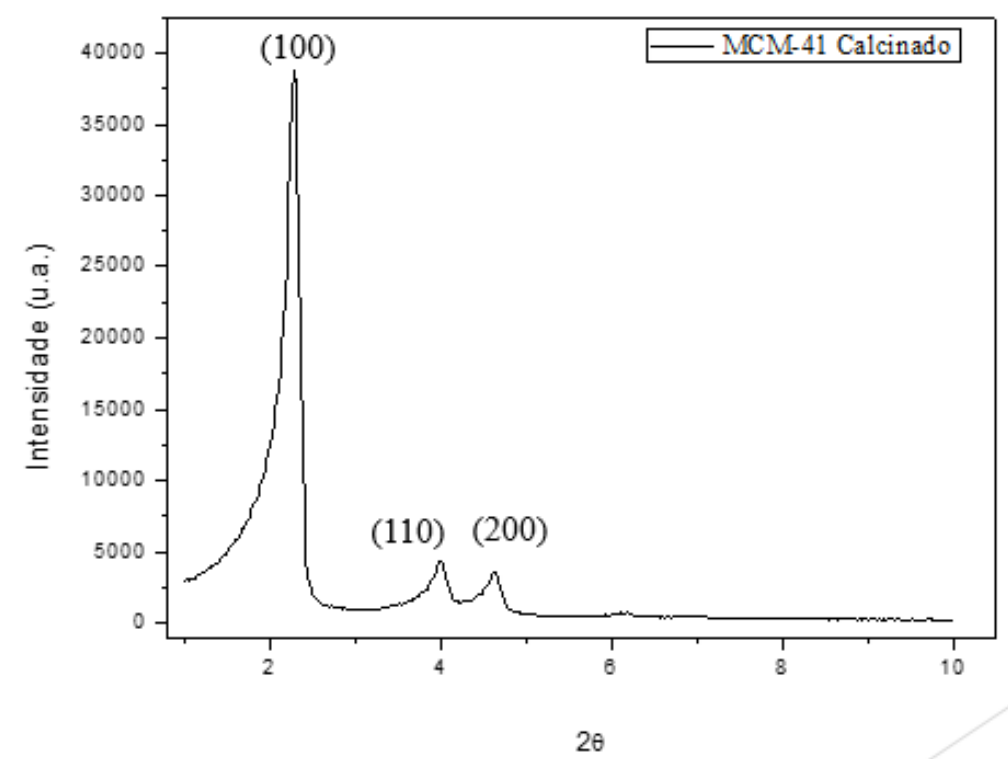

Figura 4. DRX do catalisador MCM-41 calcinado

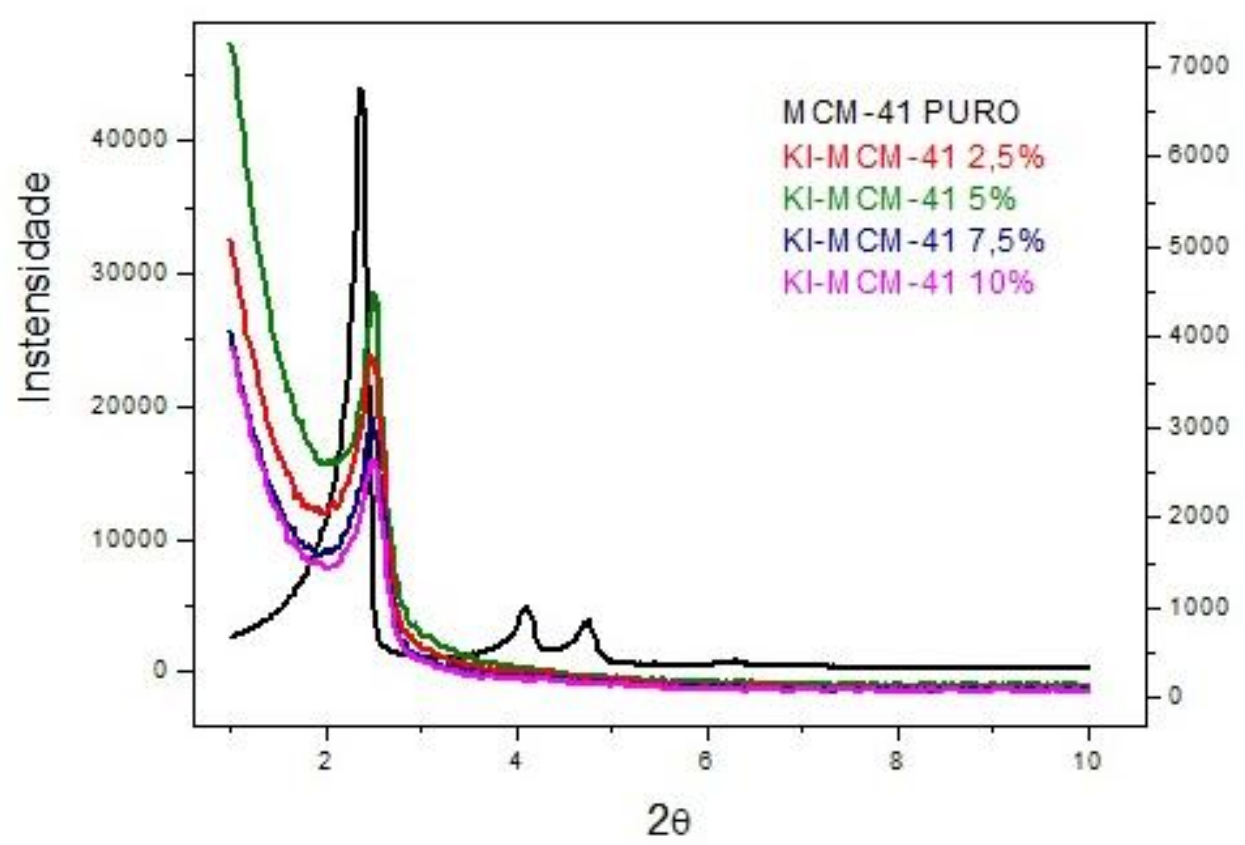

Figura 5. $D R X$ do $M C M-41$ e amostras impregnadas 


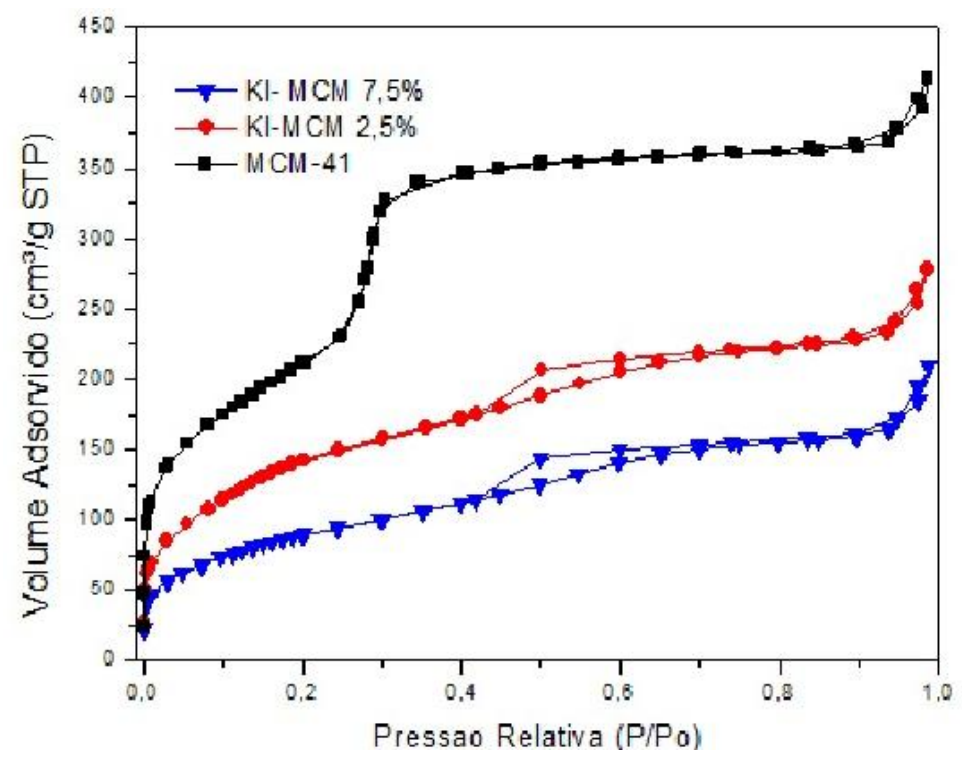

Figura 6. Curvas de adsorção-dessorção do material puro e impregnados com KI

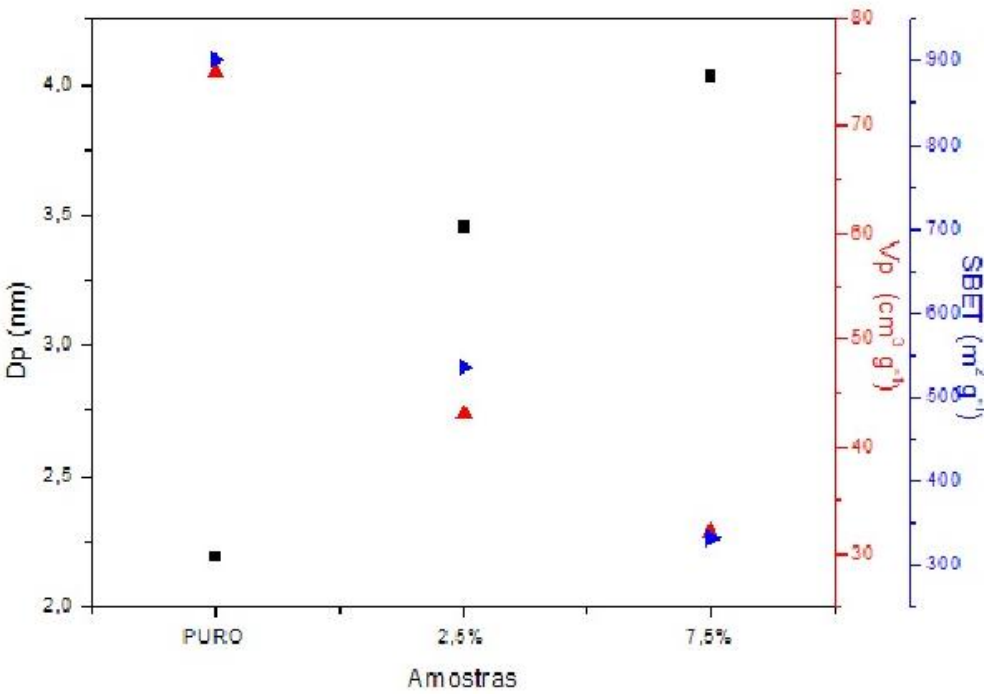

Figura 7. Propriedades texturais dos materiais obtidos 

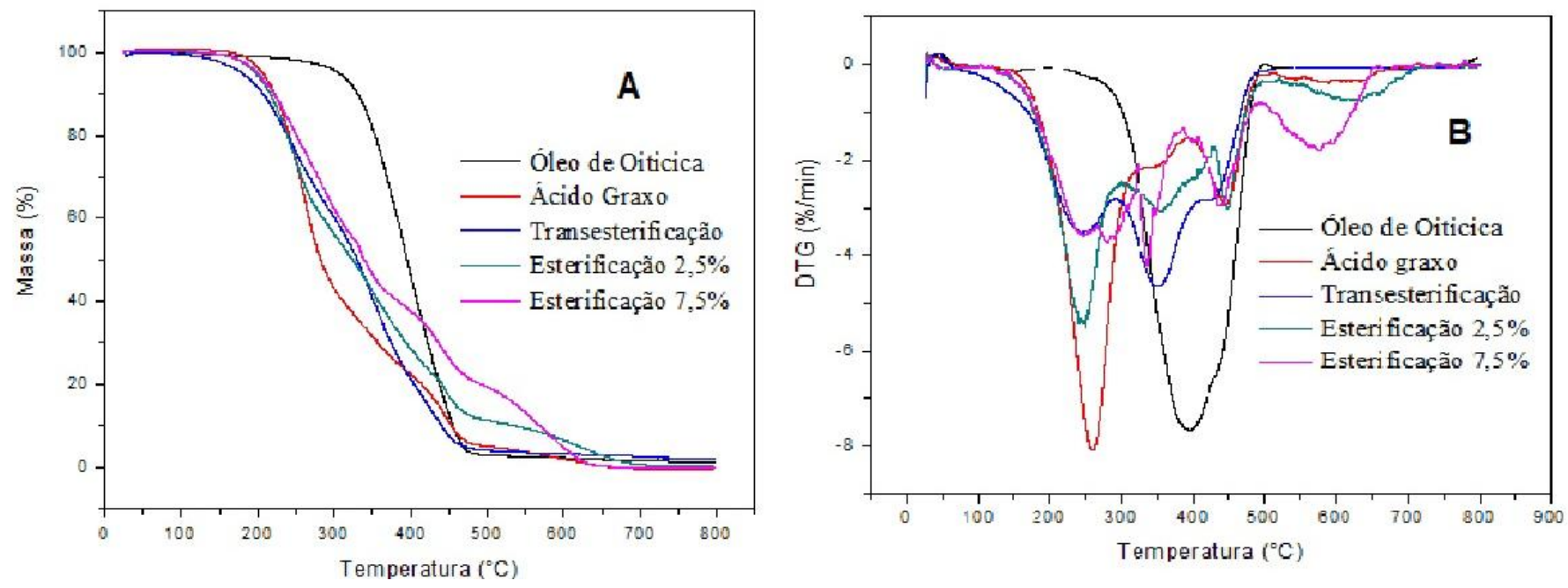

Figura 8. A e B - Curvas TG-DTG do óleo, ácido graxo, transesterificação e esterificação com 2.5 e $7.5 \%$ de $K I$

PERIÓDICO TCHÊ QUÍMICA • www.periodico.tchequimica.com • Vol. 16 N. 31

- ISSN 1806-0374 (impresso) • ISSN 1806-9827 (CD-ROM) • ISSN 2179-0302 (meio eletrônico) (C) 2019. Porto Alegre, RS. Brasil

The Periódico Tchê Química (ISSN: 1806-0374; 2179-0302) is an open-access journal since 2004. Journal DOI: 10.52571/PTQ. http://www.tchequimica.com. This text was introduced in this file in 2021 for compliance reasons.

() The Author(s)

OPEN ACCESS. This article is licensed under a Creative Commons Attribution 4.0 (CC BY 4.0) International License, which permits use, sharing, adaptation, distribution, and reproduction in any medium or format, as long in the article 's Creative Commons license unless indicated otherwise in a credit line to the material. If material is not included in the article's Creative Commons license and your intended use is not permitted by statutory regulation or exceeds the permitted use, you will need to obtain permission directly from the copyright holder. To view a copy of this license, visit http://creativecommons.org/licenses/by/4.0/. 